\title{
Portal corporativo: conceitos e características
}

\section{Cláudia Augusto Dias}

Mestranda em ciência da informação na Universidade de Brasília $(\mathrm{UnB})$. Formada em engenharia elétrica pela Universidade de Brasília. Trabalha como auditora de sistemas no Tribunal de Contas da União (TCU). É responsável pela publicação Procedimentos de Auditoria de Sistemas, participa do Projeto de Pesquisa e Desenvolvimento da Tecnologia da Informação do TCU e realiza auditorias de sistemas em empresas e órgãos da administração pública federal.

E-mail: claudiaad@yahoo.com

\section{Resumo}

Conceitos a respeito de uma nova tecnologia conhecida como portal corporativo, cuja proposta é facilitar o acesso às informações digitais no contexto organizacional. Este artigo apresenta definições, características básicas, arquitetura e tipos de portais corporativos identificados na literatura especializada, constituindo-se em parte da revisão de literatura de projeto de pesquisa em andamento, cujo objetivo geral é analisar métodos para avaliação de usabilidade de portais corporativos.

\section{Palavras-chave}

Portal corporativo; Informação institucional; Gestão da informação.

\section{Corporative gateway [portal]: concepts and characteristics}

\author{
Abstract \\ Concepts of a new technology known as corporate portal, \\ whose purpose is to provide easy access to enterprise digital \\ information. This paper presents definitions, main \\ characteristics, architecture and different kinds of corporate \\ portals found in specialized literature, being part of the \\ literature review of an ongoing research project, whose main \\ objective is to propose a usability evaluation methodology \\ applied to corporate portals.
}

\section{Keywords}

Corporate portal; Enterprise information; Information management.

\section{INTRODUÇÃO}

Com os inúmeros avanços tecnológicos, muitas instituições sofrem com o excesso de informações, sendo imprescindível a aplicação da gestão da informação para administrar esse caos informacional do mundo digital. Muitas vezes as informações estão armazenadas em equipamentos de informática de forma não integrada, espalhadas em seus bancos de dados, dificultando seu acesso e, conseqüentemente, o desempenho das atividades necessárias ao pleno funcionamento da instituição. Devido à proliferação de arquivos eletrônicos produzidos individualmente pelos funcionários, sem o intuito de compartilhamento de informações, e de várias aplicações de bancos de dados, desenvolvidas ao longo do tempo para atender a demandas específicas, falta, a essas instituições, uma visão global de seus próprios dados e informações.

Recentemente, surgiu um novo conceito, o portal corporativo, que utiliza metadados ${ }^{1}$ e a linguagem XML (Extensible Markup Language) ${ }^{2}$ para integrar dados não estruturados aos dados estruturados dos bancos de dados institucionais, fornecendo acesso às informações a partir de uma interface individualizada, disponível na rede hipertextual corporativa - Intranet ${ }^{3}$. $O$ portal corporativo é tido, por Collins, como o mais importante projeto de gestão da informação da próxima década [08]. Como uma evolução do data warehouse ${ }^{4}$, o portal corporativo estende sua aplicação à Intranet e se constitui em um único ponto de acesso a todos os recursos de informação e conhecimento em uma instituição.

Embora seja uma tecnologia muito recente, vários são os benefícios, apontados por fornecedores e consultores de informática, associados aos portais corporativos. Dentre esses benefícios, destaca-se a facilidade de acesso às informações distribuídas nos diversos sistemas, arquivos e bases de dados institucionais.

Para conseguir concretizar esse benefício, é fundamental que o projeto do portal corporativo leve em consideração a interação dos usuários com sua interface. Sua capacidade de facilitar o acesso dos usuários às informações institucionais está intrinsecamente relacionada à facilidade de uso, aprendizado e satisfação do usuário, isto é, à usabilidade de sua interface web. 
O termo usabilidade foi definido na norma ISO/IEC 9126, sobre qualidade de software, como "um conjunto de atributos de software relacionado ao esforço necessário para seu uso e para o julgamento individual de tal uso por determinado conjunto de usuários." [30] A partir de então, esse termo passou a fazer parte do vocabulário técnico de várias áreas do conhecimento, tais como tecnologia da informação, ergonomia, interação homemcomputador e psicologia aplicada, tendo sido traduzido literalmente para diversos idiomas. O conceito de usabilidade evoluiu e foi redefinido na norma ISO 9241-11 Guidelines on Usability como "a capacidade de um produto ser usado por usuários específicos para atingir objetivos específicos com eficácia, eficiência e satisfação em um contexto específico de uso." [31]

Como ferramentas auxiliares de projeto de sistemas centrado no usuário, existem vários métodos de avaliação de usabilidade, cujos objetivos são verificar a eficiência e a eficácia da interação usuário-computador durante a realização de tarefas; e identificar, de forma subjetiva, o grau de satisfação dos usuários com o sistema.

A variedade de métodos de avaliação de usabilidade existentes, sua necessidade de adaptação ao contexto de uso e a evolução tecnológica da gestão da informação, representada pelo portal corporativo, deram origem a um problema de pesquisa, relacionado à identificação e adaptação de métodos de avaliação de usabilidade para o contexto de portais corporativos.

Este artigo constitui parte da revisão de literatura de projeto de pesquisa em andamento, cujo objetivo geral é analisar métodos para avaliação de usabilidade de portais corporativos. A partir de um estudo de caso, os principais métodos serão identificados, selecionados, adaptados ao contexto tecnológico e institucional e, também, combinados em uma metodologia de avaliação de usabilidade de portais corporativos, a qual poderá ser aplicada em outras instituições, tomando como base os resultados desse estudo.

\section{DEFINIÇÃO DE PORTAL CORPORATIVO}

Há três ou quatro anos, o que hoje é chamado de portal era conhecido como máquina de busca, cujo objetivo era facilitar o acesso às informações contidas em documentos espalhados pela Internet. Inicialmente, as máquinas de busca possibilitavam ao usuário da Internet localizar documentos a partir de pesquisas booleanas ${ }^{5}$ e navegação associativa entre $\operatorname{links}^{6}$. Para reduzir ainda mais o tempo de busca na Internet e auxiliar os usuários menos experientes, vários sites ${ }^{7}$ de busca incluíram categorias, isto é, passaram a filtrar sites e documentos em grupos pré-configurados de acordo com seu conteúdo - esportes, meteorologia, turismo, finanças, notícias, cultura etc. $\mathrm{O}$ passo seguinte foi a integração de outras funções, como, por exemplo, as comunidades virtuais e suas listas de discussão, chats $^{8}$ em tempo real, possibilidade de personalização dos sites de busca (My Yahoo!, My Excite etc.) e acesso a conteúdos especializados e comerciais. Essa nova concepção de máquina de busca é que passou a ser chamada de portal.

Reynolds \& Koulopoulos identificam as seguintes fases do progresso do portal web: pesquisa booleana, navegação por categorias, personalização e, por fim, funções expandidas para outras áreas dos mundos informacionais e comerciais [40]. Essa evolução dos portais web, ou portais públicos, chamou a atenção da comunidade corporativa, a qual vislumbrou a possibilidade de utilização dessa mesma tecnologia para organizar e facilitar o acesso às informações internas da empresa.

Por ser um conceito muito recente, a terminologia relacionada com os portais corporativos ainda não se estabilizou. Os termos "portal corporativo", "portal de informações corporativas", "portal de negócios" e "portal de informações empresariais" são utilizados na literatura, algumas vezes, como sinônimos. Segundo Firestone, o processo de definição do portal corporativo, como qualquer outra estratégia de negócios, é um processo político, isto é, uma tentativa de persuadir os usuários e os investidores da área de tecnologia da informação que uma definição é mais adequada do que outra, favorecendo os interesses de um ou outro fornecedor/consultor [25]. A aceitação geral de uma definição feita por determinado fornecedor pode levar o público a concluir que o produto da concorrência, por deixar de ter esta ou aquela característica, não é realmente um portal corporativo, por exemplo. Deixando de lado o aspecto político entre concorrentes e enfatizando as características inerentes de cada termo, a seguir serão discutidas e agrupadas as definições consideradas mais relevantes para esta revisão de literatura.

O termo "portal de informações empresariais" foi utilizado pela primeira vez em um relatório da empresa de consultoria Merril Lynch, elaborado por Shilakes \& Tylman em novembro de 1998, onde consta a seguinte definição:

"Portais de informações empresariais são aplicativos que permitem às empresas libertar informações armazenadas interna e externamente, provendo aos usuários uma única 


\section{Cláudia Augusto Dias}

via de acesso à informação personalizada necessária para a tomada de decisões de negócios." [41]

Nesse relatório, o portal de informações empresariais ou EIP (Enterprise Information Portal) é considerado uma oportunidade emergente de mercado, uma estratégia de negócios, constituindo-se em um conjunto de aplicativos de software que consolida, gerencia, analisa e distribui informações não só internamente, como também para o ambiente externo à organização, incluindo ferramentas de inteligência de negócios, gestão de conteúdo, data warehouse, gestão de dados e informações [41]. Apesar de essa definição ser abrangente o suficiente para aliar duas funções dos portais corporativos, suporte à decisão e processamento cooperativo, o relatório, como um todo, não dá ênfase suficiente ao aspecto cooperativo, apesar de mencioná-lo.

Nessa mesma linha de raciocínio, White define o EIP como uma ferramenta que provê, aos usuários de negócios, uma única interface web às informações corporativas espalhadas pela empresa [49]. Dentro desse conceito mais genérico, White ressalta as duas funções mencionadas anteriormente, subdividindo os EIPs em duas categorias : "EIP para processamento de decisões" e "EIP para processamento cooperativo" [49].

Para White, o EIP para processamento de decisões auxilia executivos, gerentes e analistas de negócios no acesso às informações necessárias para a tomada de decisões de negócios, enquanto o EIP para processamento cooperativo organiza e compartilha informações de grupos de trabalho, tais como mensagens de correio eletrônico, relatórios, memorandos, atas de reunião etc. [49].

Eckerson, por sua vez, utiliza outro termo - portal de negócios - e o define como um aplicativo capaz de proporcionar aos usuários um único ponto de acesso a qualquer informação necessária aos negócios, esteja ela dentro ou fora da corporação. Eckerson compara o portal de negócios a um "shopping center para trabalhadores do conhecimento", explicando que a maioria dos consumidores prefere fazer suas compras em um shopping, por ter certeza de lá poder encontrar tudo de que precisa, evitando a busca cansativa em sucessivas lojas em diferentes localidades [15]. Sua concepção difere do ponto de vista de White, Shilakes \& Tylman por dar pouca ou quase nenhuma ênfase ao aspecto cooperativo, limitando o conceito de portal como uma via de acesso às informações estruturadas e não estruturadas, por meio de uma interface web [15].
Uma linha de pensamento um pouco diferente, defendida por Murray [34], considera o portal corporativo mais do que uma via de acesso às informações corporativas, como definem Shilakes \& Tylman [41]. Murray afirma que os portais voltados apenas para o conteúdo são inadequados ao mercado corporativo e que "os portais corporativos devem nos conectar não apenas a tudo de que necessitamos, mas a todos que necessitamos, e proporcionar todas as ferramentas necessárias para que possamos trabalhar juntos" [34]. Murray identifica quatro tipos distintos de portais empresariais: "portais de informações", "portais cooperativos", "portais de especialistas" e "portais do conhecimento" [34]. Os portais de informações provêm acesso à informação; os portais cooperativos fornecem ferramentas de processamento cooperativo; os portais de especialistas conectam pessoas, com base em suas experiências e interesses; finalmente, os portais do conhecimento combinam todas as características dos anteriores [34].

Com essa subdivisão, Murray parece estar mais interessado em um portal capaz de atender a todas as expectativas funcionais dos usuários corporativos, e não apenas em uma ferramenta de tomada de decisão ou de acesso a informações.

Assim como Murray destaca o caráter cooperativo do portal, Reynolds \& Koulopoulos vislumbram o portal como um sistema de informações centrado no usuário, integrando e divulgando conhecimentos e experiências de indivíduos e equipes, atendendo, assim, aos padrões atuais de instituições baseadas no conhecimento [40]. Para esses autores, o portal corporativo é capaz de aliar o conhecimento explícito contido em arquivos, bases de dados, correspondências, páginas web e aplicativos empresariais ao conhecimento tácito dos times de projeto, das heurísticas profissionais e das comunidades institucionais [40].

Da mesma forma que acontece com os consultores e analistas de mercado na área de informática, também os fabricantes e fornecedores de software definem os portais corporativos de maneiras diferentes, de acordo com o enfoque de cada produto, destacando características relacionadas ao suporte à decisão e/ou ao processamento cooperativo. Como concorrentes no mercado, cada fabricante realça as qualidades de seu produto em suas definições. Como exemplos, nos parágrafos seguintes são discutidas as definições de portais dadas por três conhecidos fornecedores. 
A Viador define os portais de informações empresariais como "aplicativos que possibilitam às companhias fornecer acesso às informações armazenadas interna e externamente e, ainda, oferecerem aos usuários internos e externos uma única janela para as informações personalizadas necessárias para a tomada de decisões de negócios." [45] Apesar de usar a nomenclatura EIP, considerada mais genérica, a Viador destaca claramente o suporte à tomada de decisões como a principal função de seu portal.

A Brio, por outro lado, baseando-se nos requisitos considerados pelo Patricia Seybold Group como essenciais para os portais corporativos, define seu portal de informações empresariais como uma ferramenta que provê, aos usuários, amplo acesso à informação, independentemente de onde esta esteja armazenada, e compartilhamento cooperativo de informações entre todos os usuários da organização, independentemente da localidade em que se encontram [03]. O uso da nomenclatura EIP pela Brio é compatível com as definições de White e Shilakes \& Tylman.

A Plumtree Software, por sua vez, define seu portal corporativo como um sistema capaz de reunir, em uma única página web personalizada, todas as informações e ferramentas de produtividade relevantes aos usuários corporativos, integrando ainda aplicativos dinâmicos, tais como relatórios on-line, correio eletrônico, agendas e serviços comerciais [39]. A incorporação de aplicativos cooperativos e a capacidade intrínseca do portal de criar relacionamentos entre informações e prover acesso ao conteúdo da rede corporativa e da Internet, por meio de uma visão lógica de informações provenientes de sistemas diversos, dão ao portal da Plumtree Software um caráter mais genérico, isto é, um portal capaz de suportar tanto o processamento cooperativo como o processamento de decisões. A nomenclatura portal corporativo demonstra a preocupação da Plumtree Software em diferenciar seu produto dos portais públicos, como Yahoo! e Excite.

Dada a diversidade de nomenclaturas e definições, é conveniente classificar os portais para melhor entender suas características e os enfoques dados por cada autor ou fornecedor citado.

\section{DIFERENTES TIPOS DE PORTAIS}

Há duas formas de classificar os portais: uma em relação ao contexto de sua utilização (público ou corporativo) e outra em relação às suas funções (suporte à decisão e/ou processamento cooperativo). Como o interesse desta revisão de literatura é o portal corporativo, a classificação quanto à função tomará como base o ambiente institucional.

\section{Quanto ao contexto}

Apesar das semelhanças tecnológicas, os portais públicos e os portais corporativos atendem a grupos de usuários diversos e têm propósitos completamente diferentes.

\section{Portal público}

O portal público, também denominado portal Internet, portal web ou portal de consumidores, provê ao consumidor uma única interface à imensa rede de servidores que compõem a Internet. Sua função é atrair, para o seu site, o público em geral que navega na Internet. Quanto maior o número de visitantes, maior a probabilidade do estabelecimento de comunidades virtuais que potencialmente comprarão o que os anunciantes daquele site têm para vender. Assim como a televisão, o rádio e a mídia impressa, o portal público estabelece um relacionamento unidirecional com seus visitantes e constitui-se em uma mídia adicional para o marketing de produtos.

Segundo Eckerson, desde meados de 1990, os portais públicos passaram por três gerações diferentes [16], como mostra o quadro 1 , na página seguinte.

\section{Portal corporativo}

No mundo institucional, o portal tem o propósito de expor e fornecer informações específicas de negócio, dentro de determinado contexto, auxiliando os usuários de sistemas informatizados corporativos a encontrar as informações de que precisam para fazer frente aos concorrentes [40]. O portal corporativo é considerado por Reynolds \& Koulopoulos como uma evolução do uso das Intranets, incorporando, a essa tecnologia, novas ferramentas que possibilitam identificação, captura, armazenamento, recuperação e distribuição de grandes quantidades de informações de múltiplas fontes, internas e externas, para os indivíduos e equipes de uma instituição [40].

Assim como os portais públicos, os portais corporativos também passaram por estágios evolutivos, porém em um espaço de tempo bem menor. Eckerson identifica quatro gerações de portais corporativos e considera que, no ano de 1999, os portais disponíveis no mercado passaram da primeira à terceira geração. Além disso, Eckerson acredita que os portais corporativos têm um potencial de expansão ainda maior que os portais públicos [16]. Quadro 2, a seguir. 
QUADRO 1

Gerações dos portais públicos (quadro baseado nas gerações identificadas por Eckerson [16]).

\begin{tabular}{lll}
\hline Geração & Categoria & \multicolumn{1}{c}{ Características das gerações dos portais públicos } \\
\hline Primeira & Referencial & $\begin{array}{l}\text { Máquina de busca, com catálogo hierárquico de conteúdo da web. Cada entrada do catálogo } \\
\text { contém uma descrição do conteúdo e um link. }\end{array}$ \\
Segunda & Personalizado & $\begin{array}{l}\text { O usuário, por meio de um identificador e uma senha, pode criar uma visão personalizada do } \\
\text { conteúdo do portal, conhecida como "MinhaPágina". Essa visão mostra apenas as categorias } \\
\text { que interessam a cada usuário. O portal pode avisar ao usuário sempre que um novo conteú- } \\
\text { do for adicionado às categorias por ele assinaladas. }\end{array}$ \\
Terceira & Interativo & $\begin{array}{l}\text { O portal incorpora aplicativos, tais como correio eletrônico, chat, listas de discussão, cota- } \\
\text { ção da bolsa, comércio eletrônico, leilóes, permitindo ao usuário interagir com o portal e } \\
\text { com seu provedor de conteúdo. Os usuários podem selecionar essas aplicações para suas } \\
\text { páginas pessoais. }\end{array}$ \\
\hline
\end{tabular}

QUADRO 2

Gerações dos portais corporativos (quadro baseado nas gerações identificadas por Eckerson [16]).

\begin{tabular}{|c|c|c|}
\hline Geração & Categoria & Características das gerações dos portais corporativos \\
\hline Primeira & Referencial & $\begin{array}{l}\text { Máquina de busca, com catálogo hierárquico de conteúdo da web. Cada entrada do catálogo } \\
\text { contém uma descrição do conteúdo e um link. Essa geração enfatiza mais a gerência de } \\
\text { conteúdo, disseminação em massa das informações corporativas e o suporte à decisão. }\end{array}$ \\
\hline Segunda & Personalizado & $\begin{array}{l}\text { O usuário, por meio de um identificador e uma senha, pode criar uma visão personalizada do } \\
\text { conteúdo do portal, conhecida como "MinhaPágina". Essa visão mostra apenas as categorias } \\
\text { que interessam a cada usuário. O portal pode avisar ao usuário sempre que um novo conteú- } \\
\text { do for adicionado às categorias por ele assinaladas. Os usuários podem publicar documentos } \\
\text { no repositório corporativo para que esses sejam também visualizados por outros usuários. } \\
\text { Essa geração privilegia a distribuição personalizada de conteúdo. }\end{array}$ \\
\hline Terceira & Interativo & $\begin{array}{l}\text { O portal incorpora aplicativos que melhoram a produtividade das pessoas e equipes, tais } \\
\text { como correio eletrônico, calendários, agendas, fluxos de atividades, gerência de projeto, } \\
\text { relatórios de despesas, viagens, indicadores de produtividade etc. Essa geração adiciona o } \\
\text { caráter cooperativo ao portal, provendo múltiplos tipos de serviços interativos. }\end{array}$ \\
\hline Quarta & Especializado & $\begin{array}{l}\text { Portais baseados em funções profissionais, para gerência de atividades específicas na institui- } \\
\text { ção, tais como vendas, finanças, recursos humanos etc. Essa geração envolve a integração de } \\
\text { aplicativos corporativos com o portal, de forma que os usuários possam executar transações, } \\
\text { ler, gravar e atualizar os dados corporativos, e ainda incorpora outras possibilidades como } \\
\text { comércio eletrônico, por exemplo. }\end{array}$ \\
\hline
\end{tabular}

\section{Quanto à função}

As funções mais importantes de um portal corporativo são suporte à decisão e processamento cooperativo. Alguns autores e fornecedores dão mais ênfase a uma ou outra função e costumam denominar os portais de acordo com sua principal utilização na empresa. O uso de certas nomenclaturas por parte dos fabricantes, entretanto, pode levar a interpretações equivocadas sobre as reais características de seus produtos.

\section{Portais com ênfase em suporte à decisão}

Os portais com ênfase em suporte à decisão auxiliam executivos, gerentes e analistas de negócios a acessar as informações corporativas para a tomada de decisões de negócio. Por darem pouca ou quase nenhuma ênfase ao processamento cooperativo, podem ser incluídos, nessa categoria, o portal de informações de Murray, o portal de negócios de Eckerson e o EIP para processamento de decisões de White. Levando em consideração as características destacadas pela Viador, em seu artigo [45], seu portal de informações empresariais pode ser 
considerado um exemplo de portal com ênfase em suporte à decisão.

\section{- Portal de informações ou de conteúdo}

Murray conceitua portal de informações como aquele capaz apenas de organizar grandes acervos de conteúdo a partir dos temas ou assuntos neles contidos, conectando as pessoas às informações [34]. Nessa categoria Murray inclui as máquinas de busca (internas e/ou externas) e os portais públicos. Nesse tipo de portal não há preocupação com a interatividade e o processamento cooperativo entre usuários e especialistas. Para Murray, no contexto organizacional, a aplicação de um portal apenas de conteúdo seria insuficiente para atender aos objetivos de negócios das empresas [34].

White denomina essa forma básica de portal corporativo como "portal Intranet", o qual inclui links para informações e sites web dentro e fora da organização. Assim como Murray, White considera o portal Intranet análogo aos portais públicos como o Yahoo!, por exemplo [47].

\section{- Portal de negócios}

Eckerson utiliza o termo portal de negócios como o equivalente corporativo dos portais comerciais da Internet, tais como Yahoo! e Excite [15]. Esse tipo de portal tem como função tornar disponíveis, aos usuários corporativos, informações necessárias para a tomada de decisões de negócios da instituição, tais como relatórios, pesquisas, documentos textuais, planilhas, mensagens de correio eletrônico, páginas web, vídeos etc. Segundo Davydov, o portal de negócios é o ponto de partida central para os aplicativos de gerência de conteúdo e de processamento de decisões, conectando-os a informações estruturadas e não estruturadas [10].

\section{- Portal de suporte à decisão}

O portal de suporte à decisão, na concepção de White, permite que "os usuários organizem e encontrem informações corporativas em um conjunto de sistemas que constituem a cadeia produtiva de informações de negócios." [49] Esse tipo de portal utiliza ferramentas inteligentes e aplicativos analíticos para capturar informações ${ }^{9}$ armazenadas em bases de dados operacionais, no data warehouse corporativo ou ainda em sistemas externos à organização e, a partir dessas informações, gerar relatórios e análises de negócio para serem distribuídos eletronicamente aos diversos níveis de tomada de decisão na empresa. As informações fornecidas por um portal de suporte à decisão podem ser apresentadas sob a forma de relatórios, gráficos, indicadores de desempenho sobre as operações de negócios etc., podendo ser resumidas ou detalhadas de acordo com o nível estratégico, tático ou operacional do tomador de decisão.

\section{Portais com ênfase em processamento cooperativo}

Os portais com ênfase em processamento cooperativo, assim como os sistemas groupware e de automação de escritórios, lidam com informações tanto da cadeia produtiva tradicional, armazenadas e manipuladas por aplicativos corporativos, como informações geradas por grupos ou indivíduos fora dessa cadeia. Essa categoria de portal, até o momento, foi a que menos se desenvolveu no mercado. Integram essa classe os portais cooperativos de Reynolds \& Koulopoulos, os EIPs para processamento cooperativo de White, os portais cooperativos e de especialistas de Murray.

\section{- Portal cooperativo ou para processamento cooperativo}

Esse tipo de portal utiliza ferramentas cooperativas de trabalhos em grupo (groupware) e de fluxo de tarefas/ documentos (workflow) para prover acesso a informações geradas por indivíduos ou grupos. As informações manipuladas por esse tipo de portal são geralmente não estruturadas, personalizadas e encontram-se sob a forma de textos, memorandos, gráficos, mensagens de correio eletrônico, boletins informativos, páginas web e arquivos multimídia.

\section{- Portal de especialistas}

Na concepção de Murray, um portal, para ser completo, deve ter a capacidade de relacionar e unir pessoas com base em suas habilidades e experiência [34]. Essa é a proposta do portal de especialistas - um meio de comunicação e troca de experiências entre pessoas especializadas em determinadas áreas do conhecimento, por meio de comunicação em tempo real, educação à distância e manutenção de cadastro automático de especialistas.

\section{Portais de suporte à decisão e processamento cooperativo}

Os portais mais abrangentes, que conseguem aliar as funções de suporte à decisão e processamento cooperativo, conectam os usuários não só a todas as informações, mas também a todas as pessoas necessárias para a realização dos negócios da empresa. Em um mesmo ambiente, são consolidados aplicativos de gerência de conteúdo, processamento de decisões, groupware, 


\section{Cláudia Augusto Dias}

workflow, correio eletrônico, inteligência de negócios, sistemas especialistas etc. Nessa categoria se encontram o EIP originalmente definido por Shilakes \& Tylman, o EIP de White e o portal do conhecimento de Murray. Considerando as características descritas pelos fabricantes e a possibilidade de conexão a outros aplicativos especializados, pode-se considerar o portal corporativo da Plumtree Software e o EIP da Brio como exemplos dessa categoria.

\section{- Portal do conhecimento}

O portal do conhecimento de Murray, na verdade, é um ponto de convergência dos portais de informações, cooperativos e de especialistas, sendo capaz de implementar tudo que os outros tipos de portais implementam e de fornecer conteúdo personalizado de acordo com a atividade de cada usuário [34].

\section{- Portal de informações empresariais - EIP}

O portal de informações empresariais utiliza metadados e a linguagem XML (Extensible Markup Language) para integrar os dados não estruturados, mantidos em arquivos textuais, relatórios, mensagens de correio eletrônico, gráficos, imagens etc. aos dados estruturados das bases de dados do data warehouse, fornecendo acesso às informações institucionais a partir de uma interface individualizada, disponível na rede hipertextual corporativa - Intranet.

O EIP alia as características do portal cooperativo e do portal de suporte à decisão. Firestone [25], levando em conta a intenção quase explícita de Shilakes \& Tylman de relacionar o EIP a aplicativos cooperativos e interativos para gerenciar repositórios de conhecimento, considera o portal de informações empresariais semelhante ao portal do conhecimento de Murray. Essa semelhança, porém, depende do grau de cooperação das ferramentas implementadas no EIP e da capacidade de interação entre especialistas, trocando conhecimentos, experiências e habilidades.

Nos próximos tópicos deste artigo, para evitar confusões de nomenclaturas, será utilizado o termo "portal corporativo", em oposição a "portal público", já que não há interesse em ressaltar diferenças em relação às funções de cada tipo de portal.
FIGURA 1

Elementos de um modelo conceitual de portal de informações empresariais - adaptada de Shilakes $\&$ Tylman [41].

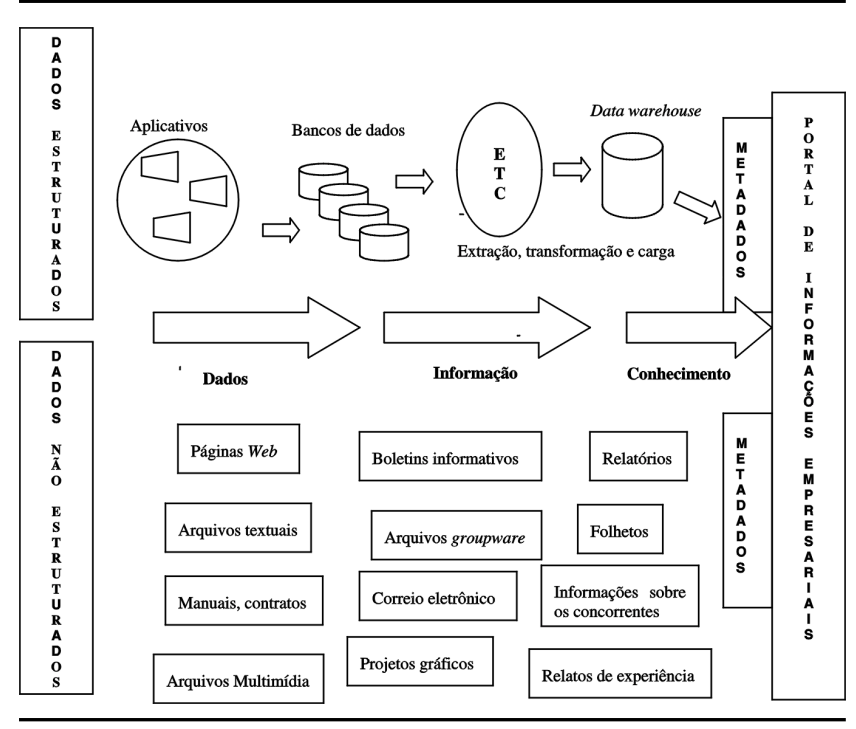

\section{REQUISITOS MÍNIMOS DE UM PORTAL CORPORATIVO}

Por congregar, em um só produto, várias tecnologias já existentes, tais como sistemas de inteligência de negócios, gestão de documentos, automação de escritórios, groupware, data warehouse, Intranet etc., os fornecedores de produtos nessas áreas têm se posicionado também como fornecedores do mercado de portais corporativos. Paralelamente, observa-se o aparecimento de pequenas empresas, novas no ramo de informática, que vislumbraram um grande potencial no mercado de portais corporativos, e ainda associações de produtos e empresas em soluções conjuntas para atender necessidades específicas de seus clientes. Com isso, a escolha de um portal corporativo, dentre os vários produtos hoje oferecidos pela indústria de informática, não é uma tarefa tão fácil como parece.

Para auxiliar os executivos das empresas nessa escolha, consultores como Eckerson, do Patricia Seybold Group, e White, do DataBase Associates International, têm publicado artigos e relatórios contendo requisitos mínimos de um portal corporativo [14, 45]. Alguns fornecedores, tais como Plumtree Software, Brio e Viador, têm utilizado esses mesmos relatórios para mostrar a conformidade de seus produtos às regras básicas neles identificadas $[03,15]$. 


\section{Portal corporativo: conceitos e características}

As 15 regras de Eckerson [14], comentadas neste quadro, resumem os principais requisitos esperados de um portal corporativo:
A esses requisitos podem ser adicionados ainda:

- habilidade de gerenciar o ciclo de vida das informações, estabelecendo níveis hierárquicos de armazenamento e descartando as informações ou documentos quando não mais necessários;

\section{QUADRO 3}

\section{Requisitos mínimos de um portal corporativo.}

\begin{tabular}{ll}
\hline \multicolumn{1}{c}{ Requisito } & \multicolumn{1}{c}{ Descrição } \\
\hline Fácil para usuários eventuais & $\begin{array}{l}\text { Os usuários devem conseguir localizar e acessar facilmente a informação correta, com o mínimo de } \\
\text { treinamento, não importando o local de armazenamento dessa informação. Encontrar informações } \\
\text { de negócios no portal deve ser tão simples quanto usar um navegador web. }\end{array}$ \\
Classificação e pesquisa intuitiva & $\begin{array}{l}\text { O portal deve ser capaz de indexar e organizar as informações da empresa. Sua máquina de busca deve } \\
\text { refinar e filtrar as informações, suportar palavras-chave e operadores booleanos, e apresentar o } \\
\text { resultado da pesquisa em categorias de fácil compreensão. }\end{array}$
\end{tabular}

Compartilhamento cooperativo

O portal deve permitir aos usuários publicar, compartilhar e receber informações de outros usuários. O portal deve prover um meio de interação entre pessoas e grupos na organização. Na publicação, o usuário deve poder especificar quais usuários e grupos terão acesso a seus documentos/objetos.

Conectividade universal aos recursos informacionais

O portal deve prover amplo acesso a todo e qualquer recurso informacional, suportando conexão com sistemas heterogêneos, tais como correio eletrônico, bancos de dados, sistemas de gestão de documentos, servidores web, groupwares, sistemas de áudio, vídeo etc. Para isso, deve ser capaz de gerenciar vários formatos de dados estruturados e não estruturados.

Acesso dinâmico aos recursos informacionais

Roteamento inteligente

Ferramenta de inteligência de negócios integrada

Arquitetura baseada em servidor

Por meio de sistemas inteligentes, o portal deve permitir o acesso dinâmico às informações nele armazenadas, fazendo com que os usuários sempre recebam informações atualizadas.

O portal deve ser capaz de direcionar automaticamente relatórios e documentos a usuários selecionados.

Para atender às necessidades de informação dos usuários, o portal deve integrar os aspectos de pesquisa, relatório e análise dos sistemas de inteligência de negócios.

Para suportar um grande número de usuários e grandes volumes de informações, serviços e sessões concorrentes, o portal deve basear-se em uma arquitetura cliente-servidor.

Serviços distribuídos

Definição flexível das permissões de acesso

Interfaces externas

Interfaces programáveis

Segurança

Para um melhor balanceamento da carga de processamento, o portal deve distribuir os serviços por vários computadores ou servidores.

O administrador do portal deve ser capaz de definir permissões de acesso para usuários e grupos da empresa, por meio dos perfis de usuário.

O portal deve ser capaz de se comunicar com outros aplicativos e sistemas.

O portal também deve ser capaz de ser "chamado" por outros aplicativos, tornando pública sua interface programável (API - Application-Programming Interface).

Para salvaguardar as informações corporativas e prevenir acessos não autorizados, o portal deve suportar serviços de segurança, como criptografia ${ }^{10}$, autenticação ${ }^{11}$, firewalls ${ }^{12}$ etc. Deve também possibilitar auditoria dos acessos a informações, das alterações de configuração etc.

Fácil administração

O portal deve prover um meio de gerenciar todas as informações corporativas e monitorar o funcionamento do portal de forma centralizada e dinâmica. Deve ser de fácil instalação, configuração e manutenção, e aproveitar, na medida do possível, a base instalada de hardware e software adquirida/ contratada anteriormente pela organização.

Customização e personalização

O administrador do portal deve ser capaz de customizá-lo de acordo com as políticas e expectativas da organização, assim como os próprios usuários devem ser capazes de personalizar sua interface para facilitar e agilizar o acesso às informações consideradas relevantes. 
- habilidade de localizar especialistas na organização, de acordo com o grau de conhecimento exigido para o desempenho de alguma tarefa;

- habilidade de satisfazer as necessidades de informação de todos os tipos de usuários da organização;

possibilidade de troca de informações com clientes, fornecedores, revendedores etc., fornecendo uma infraestrutura informacional adequada também para o comércio eletrônico.

\section{ARQUITETURA}

Cada produto disponível no mercado de portais corporativos tem características próprias, estrutura diferenciada ou componentes adicionais, apresentados como vantagens competitivas, quando comparado aos concorrentes. Entretanto, a arquitetura básica de qualquer portal corporativo segue, em linhas gerais, o modelo descrito por White [48], composto por um assistente de informações, provido por um navegador $w e b^{13}$, e um servidor $w_{e} b^{14}$ com os seguintes elementos: diretório de informações de negócios, máquina de busca, analisador de metadados, ferramenta de publicação, ferramenta de assinatura, interfaces de importação e exportação de dados. Os portais corporativos disponíveis no mercado podem ser produtos independentes ou produtos integrados em outros softwares, como é o caso de portais embutidos em groupwares, em ferramentas de inteligência de negócios ou em softwares de gestão de documentos.

\section{CONSIDERAÇÕES FINAIS}

Os portais corporativos, tendo como "ancestrais" os sistemas de suporte à decisão e os sistemas de informações gerenciais, são o próximo passo no desenvolvimento de interfaces de usuário às informações corporativas. Adaptando o ambiente organizacional para atender às necessidades dos usuários e otimizar a interação, a distribuição e a gerência dos recursos informacionais internos e externos, o portal corporativo permite que os usuários acessem as informações corporativas de forma mais ágil e personalizada, resultando, teoricamente, em aumento de produtividade, redução de custos e aumento de competitividade da organização.
FIGURA 2

Principais componentes de um portal corporativo adaptada e expandida de White [48].

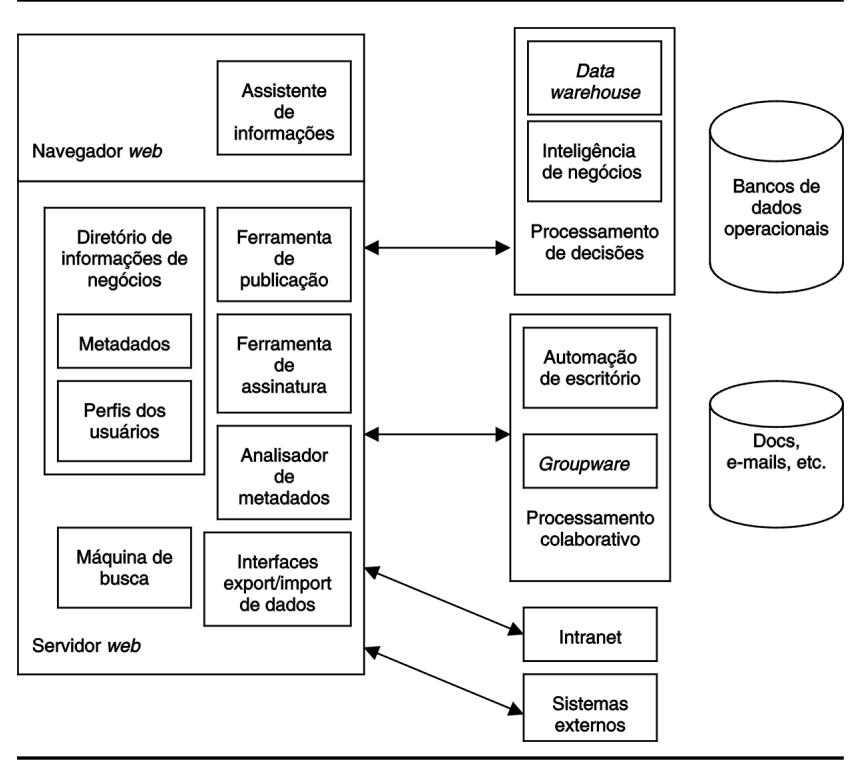

Sua capacidade de facilitar o acesso dos usuários às informações institucionais está intrinsecamente relacionada à facilidade de uso, aprendizado e satisfação do usuário com sua interface web. Para conseguir concretizar esse benefício, portanto, é fundamental que o projeto do portal corporativo leve em consideração a interação dos usuários com sua interface e tenha como objetivo minimizar seus problemas de usabilidade.

A proposta de uma metodologia de aplicação de métodos de avaliação de usabilidade, adaptada ao contexto de portais corporativos, portanto, será útil para qualquer instituição que mantenha informações sob a forma digital, disponíveis a partir da interface web de seu portal, servindo como orientação e como prova prática das vantagens e desvantagens dos métodos de avaliação utilizados na identificação de problemas de usabilidade. 


\section{Portal corporativo: conceitos e características}

\section{NOTAS}

${ }^{1}$ Descrição da estrutura, conteúdo, índices e outras propriedades dos dados, isto é, dados sobre dados.

2 Subconjunto da linguagem SGML - Standard Generalized Markup Language, sistema padronizado de organização de documentos, desenvolvido pela International Organization for Standards (ISO). A XML foi projetada para facilitar a comunicação entre sistemas conectados na Internet, provendo uma maneira fácil de definir metadados associados ao conteúdo de recursos web.

3 Aplicação da tecnologia Internet no âmbito interno da empresa.

${ }^{4}$ Grande repositório de dados, elaborado com a finalidade de dar suporte ao processo decisório estratégico da empresa.

${ }^{5}$ Pesquisas com operadores booleanos E, OU, OU EXCLUSIVO, NÃO. Com o operador E, pode-se obter, como resultado da pesquisa, todos os documentos que contenham "termo 1" E "termo 2", por exemplo. A associação de vários termos e operadores booleanos pode restringir ou ampliar o universo pesquisado.

${ }^{6}$ Ligações ou relacionamentos que apontam e conectam páginas web.

${ }^{7}$ Endereços de páginas na Internet.

${ }^{8}$ Bate-papo eletrônico feito pela Internet.

${ }^{9}$ As informações manipuladas por esse tipo de portal normalmente são estruturadas e provêm de bancos de dados.

10 "Estudo da grafia secreta, isto é, o estudo de métodos para esconder o conteúdo de mensagens ou dados armazenados. O processo de cifragem corresponde à transformação da mensagem original em algo ininteligível, utilizando um código secreto - a chave criptográfica. A decifragem, por sua vez, é o processo inverso, isto é, de recuperação da mensagem original a partir de sua forma criptografada." [13]

${ }^{11}$ Processo de identificação única de um indivíduo. Em sistemas informatizados, normalmente é feita por meio de um identificador e uma senha individual, conhecida apenas pelo usuário. $\mathrm{O}$ identificador define para o computador quem é o usuário e a senha é um autenticador, isto é, ela prova ao computador que o usuário é realmente quem ele diz ser [13].

12 "Dispositivos utilizados na proteção de redes de computadores contra ataques externos, dificultando o trânsito de invasores entre as redes." [13]

${ }^{13}$ Software que interpreta e apresenta na tela do computador as páginas web.

${ }^{14}$ Computador integrante de uma rede cliente-servidor, responsável pelos serviços relacionados com a Internet/Intranet.

\section{REFERÊNCIAS BIBLIOGRÁFICAS}

[01] ALVES, Alda J. A "revisão da bibliografia” em teses e dissertações: meus tipos inesquecíveis. Caderno de Pesquisa de São Paulo, n. 81, p. 53. 60, maio 1992.

[02] BALLA, John. Corporate portals: what they see is what they get. Intranet Journal, Jan. 2000. [online], abril 2000. [http:// www.intranetjournal.com/management/portals_021400.html].

[03] BRIO TECHNOLOGY. How to choose an enterprise information portal solution: Seybold's 10 keys to enterprise portals. Palo Alto, CA. [online], abril 2000. [http://www.brio.com/library/white_papers/pdf_files/ BrioPortal_Seybold.pdf].

[04] . Brio Portal enterprise information portal: Delivering enterprise information across the extended enterprise. Palo Alto, CA. [online], abril 2000. [http://www.brio.com/library/white_papers/ wp_brio_portal.html].

[05] BUTCHER, David \& ROWLEY, Jennifer. The 7 R's of information management. Managing Information, v. 5, n. 3, p. 34-36, Mar. 1998.

[06] CHEN, Rui. The eighth stage of information management: information resources management (IRM) vs. Knowledge management $(\mathrm{KM})$, and the chief information officer $(\mathrm{CIO})$ vs. The chief knowledge officer $(\mathrm{CKO})$. International Forum on Information and Documentation, v. 23, n. 1, p. 18-24, Jan./Mar. 1998.

[07] CHENG, Paul \& CHANG, Pintsang. Transforming corporate information into value through data warehousing and data mining. Aslib Proceedings, v. 50, n. 5, p. 109-113, May 1998.

[08] COLLINS, Daniel. Data warehouses, enterprise information portal, and the SmartMart meta directory. Information Builders Systems Journal, v. 12, n. 2, p. 53-61, Mar./Apr. 1999.

[09] CRONIN, B. \& DAVENPORT, E. Elements of information management. Scarecrow Press, 1991.

[10] DAVYDOV, Mark M. EIP: the second wave. Intelligent Enterprise, v. 3, n. 4, Mar. 2000. [online], abril 2000. [http:// www.intelligententerprise.com/000301/supplychain.shtml].

[11] DETLOR, Brian. The corporate portal as information infrastructure: towards a framework for portal design. International Journal of Information Management, v. 20, n. 2, April 2000. [online], maio 2000. [http://homepages.fis.utoronto.ca/ detlor/pubs.htm].

[12] DIAS, Cláudia. Hipertexto : evolução histórica e efeitos sociais. Ciência da Informação, v. 28, n. 3, p. 267-275, dez. 1999. [online], dezembro 1999. [http://www.ibict.br/cionline/artigos/2839905.pdf].

[13] Segurança e auditoria da tecnologia da informação. Rio de Janeiro: Axcel Books, 2000. [http://www.geocities.com/claudiaad/ publicacoes.html].

[14] ECKERSON, Wayne. 15 rules for enterprise portals. Oracle Magazine, v. 13, n. 4, p. 13-14, July/Aug. 1999. [online], abril 2000. [http:// www.oracle.com/oramag/oracle/99-Jul/49ind.html].

[15] Business portals: drivers, definitions, and rules. Boston, MA: Patricia Seybold Group, 1999. [online], abril 2000. [http:// www.viador.com/pdfs/SeyboldWhitePaper.pdf].

[16] ___ Plumtree blossoms: new version fullfills enterprise portal requirements. Boston, MA: Patricia Seybold Group, June 1999. [online], abril 2000. [http://www.plumtree.com/moreinfo/specialoffer.htm].

[17] EDMUNDS, Angela \& MORRIS, Anne. The problem of information overload in business organisations: a review of the literature. International Journal of Information Management, v. 20, n. 1, p.17-28, 2000. [online], abril 2000. [http://www.elsevier.com/cdweb/ journals/02684012/viewer.htt]. 


\section{Cláudia Augusto Dias}

[18] FERGUSON, Mike. Enterprise information portals. Information Age, Apr. 1999. [online], abril 2000. [http://www.databaseassociates.com/ pdf/April99.pdf].

[19] ___ Enterprise information portals part 2. Information Age, May 1999. [online], abril 2000. [http://www.databaseassociates.com/ pdf/May99.pdf].

[20] FINKELSTEIN, Clive. The convergence of data warehouses, Internet and Intranet technologies within enterprises. TEN - The Enterprise Newsletter, n. 6. Hillarys, WA, Australia: Information Engineering Services Pty Ltd., June 1999. [online], outubro 1999. [http:/ /www.ies.aust.com/ ieinfo/ten06.htm].

[21]___. The enterprise : XML and enterprise information portals. DM Review, July 1999. [online], outubro 1999. [http:// www.dmreview.com/master.cfm/NavID =198\&EdID =1109] .

[22] FINKELSTEIN, Clive \& AIKEN, Peter. Building corporate portals with XML. New York: McGraw-Hill, 1999. 531p.

[23] FIRESTONE, Joseph M. DKMS Brief No. Eight : Enterprise information portals and enterprise knowledge portals. [online], abril 2000. [http://www.dkms.com/EKPandEIP.html]

[24] DKMS Brief No. Ten : Benefits of enterprise information portals and corporate goals. [online], abril 2000. [http://www.dkms.com/ EIPBenefits.html].

[25] White paper No. Thirteen : Defining the enterprise information portal. July 1999. [online], abril 2000. [http://www.dkms.com/ EIPDEF.html]

[26] FITZLOFF, Emily. Portal patrol: corporate portals help early users control data deluge. Infoworld, May 17, 1999. [online], abril 2000. [http:/ /archive.infoworld.com/cgi-bin/displayStory.pl?/features/ 990517portal.htm].

[27] HORTON Jr., Forrest. Information resources management : an overview. In: Infotrends, p. 185-191, 1986.

[28] INMON, W. H. et al. Corporate information factory. New York : Wiley Computer Publishing, 1998. 274p.

[29] INMON, W. H. \& HACKATHORN, R. D. Como usar o data warehouse. Rio de Janeiro: Infobook, 1997.

[30] ISO/IEC 9126 Information technology - software product evaluation : quality characteristics and guidelines for their use. 1991

[31] ISO 9241 Part 11 Ergonomic requirements for office work with visual display terminals: Part 11 - Guidelines on usability. 1998.

[32] KELLY, Dave. Using business portals to achieve a competitive advantage. Framingham, MA: Hurwitz group, Sept. 1999. [online], abril 2000. [http://www.viador.com/pdfs/HurwitzCaseStudy.pdf].

[33] KOULOPOULOS, Tom. Corporate portals: make knowledge accessible to all. Information week online, Apr. 26, 1999. [online], abril 2000. [http://www.informationweek.com/731/31erall.htm].

[34] MURRAY, Gerry. The portal is the desktop. Intraspect, May/June 1999. [online], outubro 1999. [http://www.groupcomputing.com/ Back_Issues/1999/MayJune1999/mayjune1999.html]

[35] NIELSEN, Jakob. Intranet portals: the corporate information infrastructure, Apr. 4, 1999. [online], abril 2000. [http://www.useit.com/ alertbox/990404.html].
[36] OLIVEIRA, Djalma. Sistemas de informações gerenciais : estratégicas, táticas e operacionais. 3.ed. São Paulo : Atlas, 1996.

[37] PLUMTREE SOFTWARE. Corporate portals in brief: a simple overview of corporate portals benefits, technologies and markets. [http:/ /www.plumtree.com/moreinfo/specialoffer.htm].

[38] The currency of business-to-business commerce: syndicating gadgets from corporate portals to the world. [http://www.plumtree.com/ moreinfo/specialoffer.htm].

[39] Plumtree corporate portal 3.0. [online], abril 2000. [http:/ /www.plumtree.com/products.html].

[40] REYNOLDS, Hadley \& KOULOPOULOS, Tom. Enterprise knowledge has a face. Intelligent Enterprise, v. 2, n. 5, p. 29-34, Mar. 1999. [online], abril 2000. [http://www.intelligententerprise.com/993003/ feat $1 . s h t m l]$.

[41] SHILAKES, Christopher C. \& TYLMAN, Julie. Enterprise information portals. New York, NY: Merril Lynch, 16 Nov. 1998. [online], outubro 1999. [http://www.sagemaker.com/ home.asp?id $=500 \&$ file $=$ Company $/$ WhitePapers/lynch.htm] .

[42] SLA. - SPECIAL LIBRARY ASSOCIATION. Portals, portals, everywhere. Information outlook online. Sept. 1999. [online], abril 2000. [http://www.sla.org/pubs/serial/io/1999/sep99/edge.shtml]

[43] TAYLOR, Allan \& FARRELL, Stephen. Information management in context. Aslib proceedings, v. 44, n. 9, p. 319-322, Sept. 1992.

[44] VIADOR. Viador e-portal framework: an enterprise information portal architecture. May 2000. [online], maio 2000. [http:// www.viador.com/pdfs/Viador_Framework.pdf].

[45] Enterprise information portals: realizing the vision of 'information at your fingertips'. Jan. 1999. [online], abril 2000. [http:// www.viador.com/pdfs/EIP_white_paper_1_99.pdf].

[46] WATSON, James \& FENNER, Joe. Portals on parade. Intranet Journal, Jan. 2000. [online], abril 2000. [http://www.intranetjournal.com/ management/parade_021500.html].

[47] WHITE, Colin. Decision Threshold. Intelligent Enterprise, v. 2, n. 16, p. 35-40, Nov. 1999. [online], abril 2000. [http:// www.intelligententerprise.com/991611/feat1.shtml].

[48] _____. Enterprise information portal requirements. Decision processing brief, DP-99-02. Morgan Hill, CA: Database Associates International, Jan. 1999. [online], abril 2000. [http:// www.decisionprocessing.com/papers/eip2.doc].

[49] _. The enterprise information portal marketplace. Decision processing brief, DP-99-01. Morgan Hill, CA: Database Associates International, Jan. 1999. [online], abril 2000. [http:// www.decisionprocessing.com/papers/eip1.doc].

[50] __. Using information portals in the enterprise. DM Review, Apr. 1999. [online], outubro 1999. [http://www.dmreview.com/ master.cfm? NavID $=55 \& E d I D=61]$.

[51] WISETH, Kelli. Portal power. Oracle Magazine, v. 13, n. 6, p. 85-90, Nov./Dec. 1999. [online], fevereiro 2000. [http://www.oracle.com/oramag/ oracle/99-Nov/69ep.html]. 